\title{
The Synthesis of Glutamine-Functionalized Block Polymer and Its Application in Triple-Negative Breast Cancer Treatment
}

\author{
Yi-Zhi Zhu $\mathbb{D}^{1}{ }^{1}$ Di Xu, ${ }^{1}$ Zhen Liu $\mathbb{D},{ }^{1}$ Tian Tian, ${ }^{2}$ Fei Deng, ${ }^{1}$ Wen-Juan Tang $\mathbb{D},{ }^{1}$ Yang Wu $\mathbb{D},{ }^{1}$ \\ Wei Zhang $\left({ }^{1},{ }^{1}\right.$ and Jin-Hai Tang ${ }^{1}$ \\ ${ }^{1}$ Department of General Surgery, The First Affiliated Hospital of Nanjing Medical University, Nanjing 210029, China \\ ${ }^{2}$ School of Clinical Medicine, Xuzhou Medical University, Xuzhou 221004, China \\ Correspondence should be addressed to Wei Zhang; weizhang@njmu.edu.cn and Jin-Hai Tang; jhtang@njmu.edu.cn
}

Received 26 February 2020; Accepted 30 April 2020; Published 26 May 2020

Academic Editor: Ruibing Wang

Copyright (c) 2020 Yi-Zhi Zhu et al. This is an open access article distributed under the Creative Commons Attribution License, which permits unrestricted use, distribution, and reproduction in any medium, provided the original work is properly cited.

\begin{abstract}
Triple-negative breast cancer (TNBC) is a highly malignant tumor. At present, there are still no targeted drugs for TNBC. Clinical chemotherapeutic drugs, such as doxorubicin (DOX), have the characteristic of nontargeted distribution in treatment of TNBC, causing severe side effects. Therefore, new target treatment strategies for TNBC are of urgent need. It was speculated that glutamine could be a potential target because it is in high demand by TNBC. In this study, we found that the transporter for glutamine, ASCT2 (solute carrier family 1 member 5 (SLC1A5)), is highly expressed in TNBC by analysis of data from The Cancer Genome Atlas (TCGA) and experiments in vitro. Based on this, glutamine was grafted onto a polymeric drug carrier in order to develop a tumor-targeting drug delivery system for treatment of TNBC. Firstly, $\mathrm{pH}$-responsive glutamine-PEG $5000^{-} b-$ $\mathrm{PAE}_{10000}$ (Gln-PEG- $b$-PAE) copolymers were synthesized using Fmoc-PEG ${ }_{5000}-b$-PAE ${ }_{10000}$ (Fmoc-PEG- $b$-PAE) copolymers. Then, Gln-PEG- $b$-PAE@DOX micelles were prepared by loading DOX to Gln-PEG- $b$-PAE copolymer using a solvent casting technology. In vitro, Gln-PEG- $b$-PAE@DOX micelles exhibited $\mathrm{pH}$-dependent micellization-decellularization behavior; namely, they can rapidly release $\mathrm{DOX}$ in acidic environment of $\mathrm{pH} 6.0$ but release very slowly in physiological condition. Moreover, glutamine competition experiment showed that Gln-PEG- $b$-PAE@DOX micelles had the ability to target MDA-MB-231 cells. Compared to free DOX, Gln-PEG- $b$-PAE@DOX micelles had significantly greater cytotoxic effect and antiproliferative activity against MDA-MB-231 cells. In vivo, compared to free DOX and mPEG- $b$-PAE@DOX micelles, Gln-PEG- $b$-PAE@DOX micelles significantly inhibited tumor growth in tumor-bearing mice. Therefore, Gln-PEG-b-PAE@DOX micelles, as a tumor-targeting drug delivery system, may provide a new method for the treatment of TNBC.
\end{abstract}

\section{Introduction}

Triple-negative breast cancer (TNBC), lacking immunohistochemical expression of estrogen receptor (ER), progesterone receptor $(\mathrm{PgR})$, and human epithelial growth factor 2 (HER2), is the notorious subtype of breast cancer with highly aggressive and lethal behavior. Doxorubicin (DOX) remains one of the most standard first-line chemotherapy agents, usually used alone or in combination with other drugs for the treatment of advanced or metastatic breast cancer. However, DOX is always used limitedly due to the toxicity and some severe side effects, especially cardiotoxicity [1, 2]. Therefore, tumor-targeting drug delivery methods were studied to address this deficiency.
In recent years, more and more attention has been paid to tumor-targeting drug delivery methods to increase the availability of chemotherapeutic drugs targeting on reducing toxic side effects $[3,4]$. Polymer drug carrier is a new drug delivery technology based on pharmacology, biomaterials, and clinical medicine [5-7]. Polymeric micelles are a series of the most promising carriers to realize the precise tumor-targeted therapy and have attracted much attention in drug delivery research [8]. Polymeric micelles, formed by amphiphilic block copolymers in aqueous conditions, can form a stable nanoparticle micelle structure to encapsulate antitumor drugs and subsequently be disintegrated by specific enzyme or by varying the $\mathrm{pH}[9,10]$. The unique $\mathrm{pH}$-dependent structure of the $\mathrm{pH}$-responsive polymeric micelles is disrupted due to 
the tumoral acidic $\mathrm{pH}$, making the loaded drug more easily accumulate in tumor tissue than normal tissue $[9,11]$.

In addition to $\mathrm{pH}$-responsive polymer, the most popular tumor-targeting drug delivery strategy is to conjugate micelles with small molecule ligands, which could facilitate specific uptake by tumor cells overexpressing corresponding receptors, so as to achieve high intracellular drug dosage while minimizing nonspecific biodistribution [12, 13]. The molecule ligands include antibodies, peptides, aptamers, and phage, such as epidermal growth factor receptor (EGFR), vascular endothelial growth factor (VEGF), arginyl-glycylaspartic acid (RGD), polypeptides, and mucin 11 (MUC11) [14]. Amino acids play an important role in the biological process of living cells, especially in the proliferation and maintenance of tumor cells. Glutamine, highly taken up by TNBC cells, is a vital kind of nutrient in tumor metabolism by providing nitrogen for protein and nucleotide synthesis [15-17]. ASCT2 (solute carrier family 1 member 5 (SLC1A5)), a $\mathrm{Na}^{+}$coupled transporter for amino acid, is overexpressed in TNBC [17]. It has a high affinity for glutamine, especially at acidic pH [18]. Additionally, folic acid and methionine are frequently chosen as the targeting ligands for improving tumor-specific delivery; their comediated micelles presented higher affinity for cancer cells, compared with micelles containing folic acid or methionine alone [19]. Due to the overexpression of SLC1A5 in tumor cells, SLC1A5 was used as a target for tumor treatment. A previous study reported a competitive small molecule antagonist of transmembrane glutamine flux that selectively targets SLC1A5 to contribute to antitumor responses in vitro and in vivo [20]. Moreover, a research team discovered a targeting strategy based on polyglutamines with high affinity for SLC1A5 which can be used as a drug/gene delivery system [21].

In this study, we have prepared Gln-PEG- $b$-PAE, $\mathrm{pH}$-responsive, and glutamine-grafted copolymers as drug carriers. Then, taking advantage of high uptake of glutamine by TNBC cells, we used $\mathrm{pH}$-responsive Gln-PEG- $b$-PAE copolymer to load free DOX to achieve the purpose of targeted transport of DOX to TNBC cells. Gln-PEG- $b$-PAE@DOX micelles can target tumor cells and release DOX in tumor tissues, making it a new tumor-targeting drug delivery system for TNBC.

\section{Materials and Methods}

2.1. Data Source and Analysis. Transcriptome and clinical data was downloaded from The Cancer Genome Atlas (TCGA) data portal, including 113 normal human breast tissue samples and 5 subtypes of breast cancer with 449 luminal A, 181 luminal B, 69 HER2 positive, 166 TNBC basal-like tissue samples, and 33 TNBC normal-like tissue samples based on PAM50 intrinsic breast cancer subtypes. The RNA sequencing data of SLC1A5 were downloaded with Fragments Per Kilobase Million (FPKM). The unpaired Student's $t$-test was used to calculate the statistical significance of difference between two independent groups, and $p<0.05$ was considered statistically significant using GraphPad Prism (La Jolla, CA, USA). Patients were stratified into a high SLC1A5 expression group and a low SLC1A5 expression group based on the cut-off value of Kaplan-Meier analysis with the log-rank test between the two groups. The analysis was performed in GraphPad Prism (La Jolla, CA, USA). The hazard ratio (HR) with 95\% confidence interval (CI) was applied to report the results, and $p<0.05$ was considered statistically significant.

2.2. Cell Lines and Cell Culture. The human breast cancer cell lines, including MDA-MB-231, MCF-7, SKBR3, ZR-75-1, and BT549 cells, were acquired from Shanghai Institutes for Biological Sciences (SIBS, China). MDA-MB-231, MCF-7, and SKBR3 cells were cultured in Dulbecco's modified Eagle's medium (DMEM) (high glucose, KGM12800-500, Keygen Biotech, Nanjing, China) containing 10\% heat-inactivated fetal bovine serum (A31608-01, Gibco, NY, USA) and penicillin-streptomycin ( 80 units/mL and $80 \mu \mathrm{g} / \mathrm{mL}$ ). MCF-7 cells were cultured in the same condition, with $0.01 \mathrm{mg} / \mathrm{mL}$ human recombinant insulin (91077C-1G, Sigma-Aldrich, MO, USA) in addition. BT549 cells were cultured in DMEM containing 20\% heat-inactivated fetal bovine serum (A31608-01, Gibco, NY, USA). ZR-75-1 cells were cultured in Roswell Park Memorial Institute- (RPMI-) 1640 (KGM31800-500, Keygen Biotech, Nanjing, China) containing $10 \%$ heat-inactivated fetal bovine serum (A31608-01, Gibco, NY, USA) and penicillin-streptomycin ( 80 units $/ \mathrm{mL}$ and $80 \mu \mathrm{g} / \mathrm{mL}$ ). All the cells were incubated in a humidified atmosphere at $37^{\circ} \mathrm{C}$ under $5 \% \mathrm{CO}_{2}$.

2.3. Gene Expression Analysis. Total RNA from cell lines MDA-MB-231, MCF-7, SKBR3, ZR-75-1, and BT549 was extracted using an RNAsimple Total RNA Kit (DD419, Tiangen Biotech, Beijing, China) according to the manufacturer's instruction. The concentration and quality of the RNA were measured using the UV absorbance at 260 and $280 \mathrm{~nm}(260 / 280 \mathrm{~nm})$ on a OneDrop OD-1000+ spectrophotometer (OD-1000, OneDrop, Nanjing, China). $500 \mathrm{ng}$ of total RNA was reverse transcribed using HiScript II Q RT SuperMix for qPCR (R222-01, Vazyme, Nanjing, China) following the manufacturer's instruction. For determination of mRNA expression levels, RT-qPCR was then performed on the StepOnePlus Real-Time PCR System (4376600, Thermo Fisher Scientific, Waltham, MA, USA) with AceQ qPCR SYBR Green Master Mix (high ROX premixed) (Q141-02, Vazyme, Nanjing, China). Cycling parameters, which include a complete melt curve stage, were set up as follows: $95^{\circ} \mathrm{C}$ for $10 \mathrm{~min}$ followed by 40 cycles at $95^{\circ} \mathrm{C}$ for $10 \mathrm{~s}$ and $60^{\circ} \mathrm{C}$ for $30 \mathrm{~s}$. Primers used to amplify SLC1A 5 are as follows: forward: $5^{\prime}$-TATTTTGGCGGCTGTAGTTGTG-3' ${ }^{\prime}$; reverse: $5^{\prime}$-CCTGGGAGTGTTTCTGTTATTGTG-3'. GAPDH was used as an internal control to normalize mRNA. All the primers were purchased from RiboBio (Guangzhou, China). All the samples were analyzed in triplicate for each specific gene. The $\mathrm{Ct}$ values for each gene were normalized to the endogenous control, and the relative expression levels were calculated by the $\Delta \Delta \mathrm{Ct}$ method.

2.4. Western Blot Analysis. Total proteins from cells were extracted using radioimmunoprecipitation assay (RIPA) lysis buffer (P0013K, Beyotime, Shanghai, China) on the basis of 
the instruction. Protein content was estimated with a Multiskan FC spectrophotometer (51119050, Thermo Fisher Scientific, Waltham, MA, USA); then, proteins were mixed with sodium dodecyl sulfate-polyacrylamide gels (SDSPAGE) buffer (PG112, Yamei, Shanghai, China) and boiled for $5 \mathrm{~min}$. Equal amounts of proteins were separated by electrophoresis on 10\% SDS-PAGE and transferred to polyvinylidene difluoride (PVDF) membranes (FFP32, Merck Millipore, Darmstadt, Germany). After blocking in 5\% nonfat milk powder for $2 \mathrm{~h}$, the membranes were then probed with the following primary antibodies for another $2 \mathrm{~h}$ at room temperature: anti-SLC1A5 (1:1000) (ab84903, Abcam, Cambridgeshire, British) and anti-GAPDH $(1: 10000)$ (60004-1-Ig, ProteinTech, Chicago, IL, USA). The PVDF membranes were washed with Tween 20 (1247ML100, BioFroxx, Bensheim, Germany)/TBS (TBST) three times (10 min per wash) and incubated appropriately with a diluted horseradish peroxidase-linked secondary antibody, goat antirabbit ( $1: 1000)$ (A0208, Beyotime, Shanghai, China) and goat anti-mouse (1:1000) (A0216, Beyotime, Shanghai, China), at room temperature for $1 \mathrm{~h}$. After washing with TBST as previously described, protein bands were visualized by an enhanced chemiluminescence (ECL) plus kit (P0018S, Beyotime, Shanghai, China).

2.5. Synthesis of Gln-PEG-b-PAE Copolymer. For the purpose of designing a TNBC-targeting and $\mathrm{pH}$-responsive drug delivery system, Fmoc-PEG- $b$-PAE copolymer was synthesized by Michael addition reaction. Then, glutamine was modified to $\mathrm{NH}_{2}$-PEG- $b$-PAE copolymer through DCC/DMAP coupling chemistry. To acquire the Gln-PEG- $b$-PAE copolymer, Fmoc-PEG- $b$-PAE copolymer was synthesized as the literature described [22]. Typically, Fmoc-protected PEG (0.2 mmol, RuiXi, China) was dissolved in anhydrous dichloromethane (20 mL, Aladdin, Shanghai, China), 2 times of equivalent triethylamine was added, and the solution was placed in the reaction bottle in $0^{\circ} \mathrm{C}$ cold hydrazine (DC-3006, Shanghai Sunny Hengping Scientific Instrument, Shanghai, China). Then, acryloyl chloride $(0.4 \mathrm{mmol}$, Aladdin, Shanghai, China) was slowly added to the reaction bottle. After $2 \mathrm{~h}$ of stirring at $0^{\circ} \mathrm{C}$, the solution was recovered to room temperature and stirred for another $24 \mathrm{~h}$. The Fmocprotected poly(ethylene glycol) methyl ether acrylate (monoacylated Fmoc-PEG) was obtained by vacuuming the solution and precipitating in hexane solution. The FmocPEG poly( $\beta$-amino ester) (Fmoc-PEG- $b$-PAE) copolymer was prepared by Michael addition reaction. One equiv. of 1,6-hexanediol diacrylate (HDD, Aladdin, Shanghai, China) and 1.1 equiv. of 3 -aminopropanol (TDP, Aladdin, Shanghai, China) were dissolved in $2 \mathrm{~mL}$ of chloroform; then, 0.1 equiv. of monoacylated Fmoc- PEG $_{5000}$ was added and stirred at $50^{\circ} \mathrm{C}$ for $48 \mathrm{~h}$. The Fmoc-PEG-poly $(\beta$-amino ester $)$ copolymer was precipitated by adding an amount of methyl tertbutyl ether solution. The $\mathrm{NH}_{2}$-PEG- $b$-PAE copolymer was obtained by deprotection reaction. Briefly, Fmoc-PEG- $b$ PAE copolymer $(0.1 \mathrm{mmol})$ was dissolved in $8 \mathrm{~mL}$ of dichloromethane; then, $2 \mathrm{~mL}$ of piperidine was added and stirred at room temperature for $1 \mathrm{~h}$. The solution was decompressed and concentrated after being washed by diluted hydrochloric acid solution, and $\mathrm{NH}_{2}$-PEG- $b$-PAE copolymer was obtained by precipitation. $\mathrm{NH}_{2}$-PEG- $b$-PAE copolymer $(0.1 \mathrm{mmol})$ and Fmoc-glutamine $(0.2 \mathrm{mmol})$ were dissolved in $15 \mathrm{~mL}$ of chloroform; then, $0.2 \mathrm{mmol}$ of $\mathrm{N}, \mathrm{N}^{\prime}$-dicyclohexylcarbodiimide (DDC, Aladdin, Shanghai, China) and $0.05 \mathrm{mmol}$ of 4-dimethylaminopyridine (DMAP, Aladdin, Shanghai, China) were added into the solution to react overnight at room temperature. The Fmoc-protected Gln-PEG- $b$-PAE copolymer was collected after precipitating it in methyl tert-butyl ether solution. The Gln-PEG- $b$-PAE $(0.05 \mathrm{mmol})$ copolymer was obtained by deprotection reaction and characterized by a ${ }^{1} \mathrm{H}$ NMR and a gel permeation chromatography (GPC): ${ }^{1} \mathrm{H}$ NMR (DMSO- $d_{6}, 300 \mathrm{MHz}$ ), $\delta$ (ppm), 3.97-4.21 $\mathrm{OCH}_{2} \mathrm{CH}_{2} \mathrm{O}, 3.15-3.78 \mathrm{NHCH}_{2} \mathrm{CH}_{2} \mathrm{O}, 2.75-3.17 \mathrm{NCH}_{2} \mathrm{CH}_{2} \mathrm{O}$, $\mathrm{CHCH}_{2} \mathrm{CH}_{2} \mathrm{~N}, \quad 2.07-2.09 \mathrm{NH}_{2} \mathrm{COCH}_{2} \mathrm{CH}_{2} \mathrm{CH}, \quad 1.13-1.89$ $\mathrm{CHCH}_{2} \mathrm{CH}_{2} \mathrm{CH}_{2} \mathrm{CH}$, and $\mathrm{NCH}_{2} \mathrm{CH}_{2} \mathrm{CH}, \mathrm{CH}_{2} \mathrm{CH}_{2} \mathrm{OCO}$.

2.6. Preparation and Characterization. Gln-PEG-b-PAE@DOX micelles were prepared by loading DOX to Gln-PEG$b$-PAE copolymer using a solvent casting technology. Firstly, the dissolving DOX and the Gln-PEG- $b$-PAE copolymer were sufficiently stirred in the solution, followed by evaporation of the solvent and dissolution of the dried thin film with phosphate-buffered solution (PBS). Then, the DOX-encapsulated Gln-PEG-b-PAE micelles were manufactured from the solution by sonicating, and the encapsulation efficiency was up to $75.3 \%$.

The Gln-PEG- $b$-PAE copolymer and DOX-loaded micelles were prepared by organic solvent evaporated method. Typically, $10 \mathrm{mg}$ of Gln-PEG- $b$-PAE copolymer and $1 \mathrm{mg}$ of DOX were dissolved in chloroform in a round-bottom flask, and then, the solvent was removed by rotary evaporators. The DOX-loaded micelles were obtained by adding $5 \mathrm{~mL}$ of PBS (10 mM, pH 7.4) in the round-bottom flask which was rotated for $3 \mathrm{~h}$. After dispersing the micelles in a probe-type sonifier, the free DOX in micelle solution was removed by washing it with size exclusion centrifugal filters ( 0.5 centrifugal filters, Merck Millipore, Darmstadt, Germany, 5000 rcf, $10 \mathrm{~min}$ ) for 3 times in PBS. The empty micelles were obtained without addition of DOX. The average particle size and morphology of Gln-PEG- $b$-PAE copolymer and DOX-loaded micelles were measured by dynamic light scattering (DLS) with a particle sizing analyzer (Zeta Plus, Brookhaven, Suffolk, NY, USA) and transmission electron microscopy (TEM, FEI Tecnai F-20, USA).

The DOX loading ability of Gln-PEG- $b$-PAE copolymer was quantified by UV-Vis spectroscopy (UV-3600, Shimadzu, Kyoto, Japan) analysis. The freeze-dried DOXloaded micelles were weighed and dissolved in chloroform. The absorbance of chloroform solution at $483 \mathrm{~nm}$ was measured to determine DOX content in the micelles using an established free DOX calibration curve.

The critical micelle concentration (CMC) was measured by a fluorescence reader (Infinite 200 PRO, Tecan, Männedorf, Switzerland) using pyrene as a fluorescence probe. Briefly, the Gln-PEG- $b$-PAE copolymer was dissolved in chloroform and the concentration of the copolymer varied from $0.00187 \mathrm{mg} / \mathrm{mL}$ to $1 \mathrm{mg} / \mathrm{mL}$. Then, $100 \mu \mathrm{L}$ of pyrene $(1 \mathrm{mg} / \mathrm{L})$ was added in copolymer solution, which makes 
the volume of the mixture $1 \mathrm{~mL}$ in PBS after evaporating the organic solvent. The fluorescence intensity of copolymer was performed at $373 \mathrm{~nm}$ and $384 \mathrm{~nm}$. The CMC was estimated from the onset of a decrease in the intensity ratio of peaks at $373 \mathrm{~nm}$ to peaks at $384 \mathrm{~nm}$ plotted versus the polymer concentrations.

2.7. Cell Uptake and Tumor-Targeting Experiment. To verify whether Gln-PEG- $b$-PAE@DOX micelles could target MDA-MB-231 cells through SLC1A5 receptor-mediated transport, we added a large amount of glutamine into the medium of MDA-MB-231 cells to compete with Gln-PEG$b$-PAE@DOX micelles. Briefly, MDA-MB-231 cells in the logarithmic growth phase were plated in 6-well plates at density of $5 \times 10^{5} /$ well. After overnight culture, the cells were firstly washed by PBS solution, then added with serum-free medium, and incubated for $1 \mathrm{~h}$. Subsequently, Gln-PEG- $b$ PAE@DOX micelles were added to the well plates; meanwhile, $5 \mathrm{mg} / \mathrm{mL}$ of glutamine was added to one of the groups of cells. After $6 \mathrm{~h}$ of coculture, cells were washed by PBS for three times and visualized with an Axio Vert.A1 microscope (Carl Zeiss, Oberkochem, Germany) equipped with a fluorescence light source (X-Cite 120Q, Excelitas Technologies, Waltham, MA, USA). Moreover, MDA-MB-231 cells were harvested to measure the fluorescence intensity of the cells by FL1 channel of flow cytometry (Accuri C6, BD Biosciences, NJ, USA).

2.8. Antitumor Efficacy of Gln-PEG-b-PAE@DOX Micelles In Vitro. The antiproliferative effect of Gln-PEG- $b$-PAE@DOX micelles on MDA-MB-231 cells was analyzed by the methylthiazoltetrazolium (MTT) assay. MDA-MB-231 cells were seeded in 96-well plates at density of $8 \times 10^{3} /$ well cultured with $100 \mu \mathrm{L}$ of complete medium per well for $24 \mathrm{~h}$. Gln-PEG-b-PAE@DOX micelles and free DOX were added into wells, respectively, at different final concentrations $(0,0.625,1.25,2.5,5,10$, and $20 \mu \mathrm{g} \mathrm{DOX} / \mathrm{mL})$, and incubated for $24 \mathrm{~h}$. Meanwhile, Gln-PEG- $b$-PAE micelles and mPEG- $b$ PAE micelles were added into other wells, respectively, and incubated for $1,5,10,15,20$, and $25 \mathrm{~h}$ successively. The absorbance at $490 \mathrm{~nm}$ was eventually calculated by a Multiskan FC spectrophotometer (51119050, Thermo Fisher Scientific, Waltham, MA, USA), and the cell viability was also measured correspondingly. Flow cytometry was used to analyze the apoptosis effect of Gln-PEG- $b$-PAE@DOX micelles on MDA-MB-231 cells. MDA-MB-231 cells were cocultured with Gln-PEG-b-PAE@DOX (DOX, $1 \mathrm{mg} / \mathrm{mL})$ and free DOX $(1 \mathrm{mg} / \mathrm{mL})$ separately at the final concentration of $10 \mu \mathrm{g} D O X / \mathrm{mL}$ for $48 \mathrm{~h}$. Then, cells were collected and washed twice with $\mathrm{PBS}$ in $4^{\circ} \mathrm{C}$ and dual stained with $5 \mu \mathrm{L}$ of Annexin $\mathrm{V}$ Alexa Fluor 647 (FMSAV647-2-50, Fcmacs Biotech, Nanjing, China) and $2 \mu \mathrm{L}$ of 4',6-diamidino-2-phenylindole (DAPI solution, $1 \mathrm{mg} / \mathrm{mL}, \mathrm{BD}$ Biosciences, NJ, USA) for $15 \mathrm{~min}$ in the dark at room temperature before analysis [23]. A FACScan flow cytometer (FACSVerse, BD Biosciences, NJ, USA) was applied to analyze cellular apoptosis.
2.9. Antitumor Efficacy of Gln-PEG-b-PAE@DOX Micelles In Vivo. Subcutaneous tumors were established by inoculating $1.0 \times 10^{5}$ luciferase-labeled MDA-MB-231 (MDA-MB-231luc) cells into the right armpit of female $\mathrm{BLAB} / \mathrm{c} \mathrm{nu} / \mathrm{nu}$ mice and allowing them to grow for 14 days. When the tumor volume reached about $100 \mathrm{~mm}^{3}$, the mice were randomly assigned into 4 groups; subsequently, tumor-bearing mice (6 per group) were injected in the tail veins with the following: group 1, saline; group 2, free DOX solution $(2 \mathrm{mg} / \mathrm{kg})$; group 3, mPEG- $b$-PAE@DOX micelles (DOX $2 \mathrm{mg} / \mathrm{kg}$ ); and group 4, Gln-PEG- $b$-PAE@DOX micelles (2 mg DOX/kg) once every 4 days for 4 times. Every day, the tumor volume was calculated by a digital calliper as $a \times b^{2} / 2$, where $a$ was the largest diameter and $b$ was the smallest diameter. Five days after the last injection, the fluorescence signals of tumors were recorded by an in vivo imaging system (IVIS Spectrum, PerkinElmer, Waltham, MA, USA), using $20 \mathrm{mg} / \mathrm{mL}$ D-Luciferin Firefly potassium salt (luc001, Sciencelight Biology Science\&Technology, Shanghai, China). The experiment had previously been approved by Animal Ethical and Welfare Committee of Nanjing Medical University; the ethical approval code was IACUC-1906003.

\section{Results and Discussion}

3.1. Expression of SLC1A5 in Different Subtypes of Human Breast Cancer. Survival analysis showed that the overall survival (OS) of patients with high SLC1A5 expression was significantly lower compared to that of patients with low SLC1A5 expression $(p=0.048)$ (see Figure $1(\mathrm{a})$ ), indicating that high expression of SLC1A5 is a poor prognostic factor for breast cancer patients, making SLC1A5 a potential therapeutic target in the treatment of breast cancer. In order to investigate the expression of SLC1A5 in different subtypes of breast cancer, we firstly obtained SLC1A5 expression data and clinical information of breast cancer patients from TCGA database. SLC1A5 expression was found to be the highest in TNBC patients and significantly higher compared to normal tissues and patients with luminal A breast cancer (see Figure 1(b)). Then, we analyzed the expression level of SLC1A5 in different breast cancer cell lines including the luminal A (MCF-7), luminal B (ZR-75-1), HER2 positive (SKBR3), and TNBC (MDA-MB-231, BT549) cell lines using RT-qPCR. We observed that SLC1A5 was markedly highest in TNBC cell lines compared with other cell lines, especially in the luminal A and luminal B cell lines (see Figure 1(c)). Furthermore, Western blotting was used to compare the protein expression level of SLC1A5 in the luminal A (MCF-7), luminal B (ZR-75-1), HER2 positive (SKBR3), and TNBC (MDA-MB-231, BT549) cell lines (see Figures 1(d) and 1(e)). Consistent with PCR results, Western blotting analyses also revealed that the protein expression level of SLC1A5 in TNBC cell lines, especially in MDA-MB-231, was significantly higher than other subtypes.

A previous study revealed that SLC1A5 not only is overexpressed but also has a marked reliance on its activity for sustained cellular proliferation transporter activity in breast cancer [17]. In this study, SLC1A5 was confirmed to be overexpressed in TNBC cell lines as well as in human TNBC 


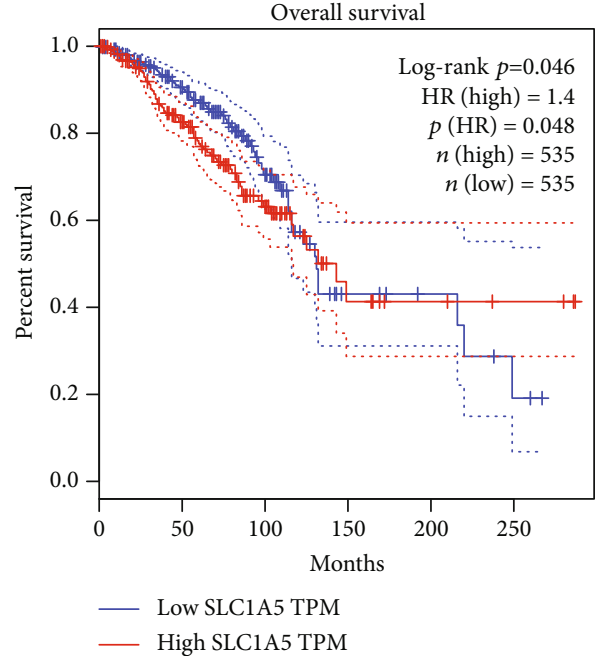

(a)

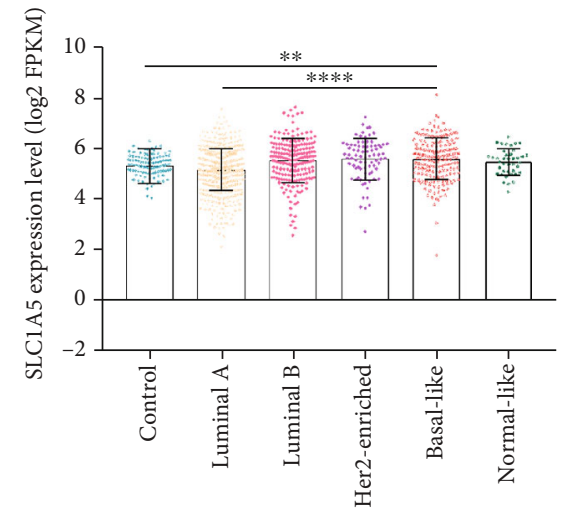

(b)

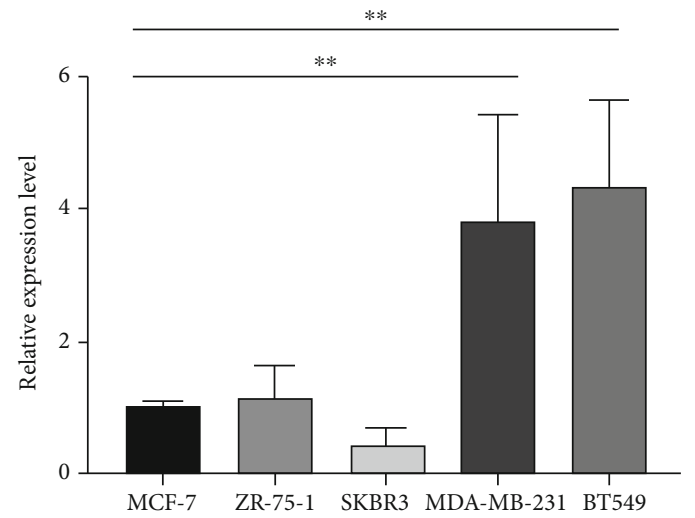

(c)

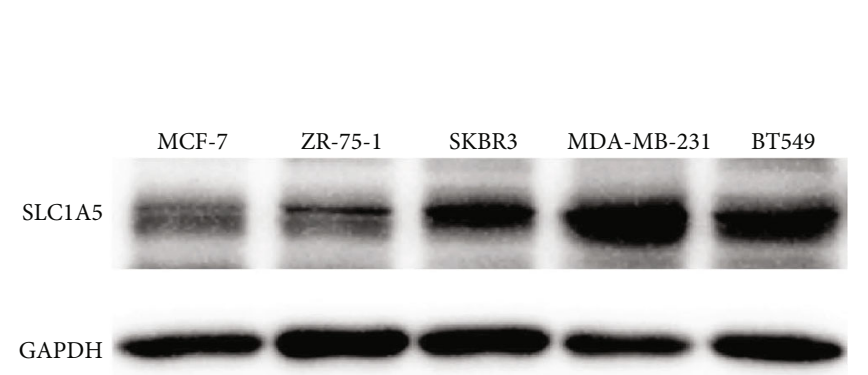

(d)

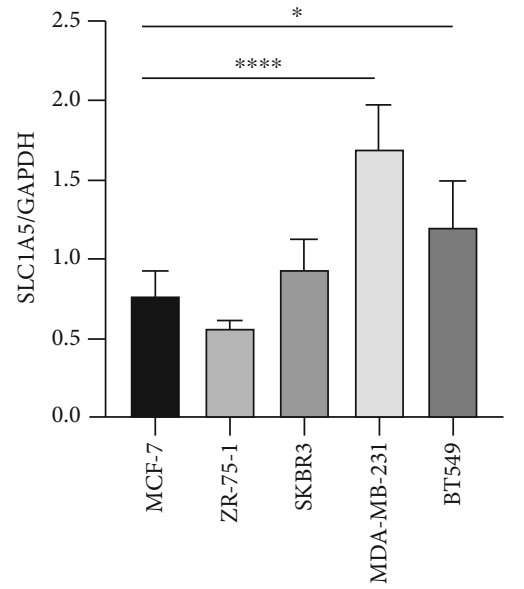

(e)

FIGURE 1: Expression of SLC1A5 in different subtypes of human breast cancer. (a) Kaplan-Meier analysis of overall survival of SLC1A5 in breast cancer. (b) The mRNA expression data of SLC1A5 downloaded from TCGA database in 113 normal human breast tissue samples and 5 subtypes of breast cancer with 449 luminal A, 181 luminal B, 69 HER2 positive, and 166 TNBC basal-like tissue samples and 33 TNBC normal-like tissue samples based on PAM50 intrinsic breast cancer subtypes; ${ }^{*} p<0.05,{ }^{* *} p<0.01$, and ${ }^{* * *} p<0.001$. (c) Relative mRNA expression of SLC1A5 in the luminal A (MCF-7), luminal B (ZR-75-1), HER2 positive (SKBR3), and highly aggressive TNBC (MDA-MB-231, BT549) cell lines. Error bars represent the mean $\pm \mathrm{SD}$ of at least three independent experiments; ${ }^{*} p<0.05,{ }^{* *} p<0.01$, and ${ }^{* * *} p<0.001$. (d, e) Protein expression of SLC1A5 in the luminal A (MCF-7), luminal B (ZR-75-1), HER2 positive (SKBR3), and highly aggressive TNBC (MDA-MB-231, BT549) cell lines. Error bars represent the mean \pm SD of at least three independent experiments; ${ }^{*} p<0.05,{ }^{* *} p<0.01$, and ${ }^{* * *} p<0.001,{ }^{* * * *} p<0.0001$. 
tissue samples compared to other subtypes by analysis of data from TCGA database. We further verified that TNBC cells highly express SLC1A5, because TNBC cells may require higher glutamine supply to provide energy for highly aggressive biological behavior. TNBC cells have a high demand for glutamine, and the receptor of glutamine on TNBC cells may be a potential target for treatment. A previous study has used glutamine analogue-modified therapeutic compound delivery systems to target glutamine-addictive cancer cells [21]. Therefore, we further explore a TNBC target therapy strategy using glutamine-grafted block copolymer as drug carrier.

3.2. Characterizations of Gln-PEG-b-PAE Copolymer. The chemical structure and molecular weight of Gln-PEG- $b$ PAE copolymer were confirmed by ${ }^{1} \mathrm{H}$ NMR (Figure 2(a)) and GPC methods, respectively. The results showed that the Gln-PEG- $b$-PAE copolymer was successfully synthesized, and the Mw of the Gln-PEG- $b$-PAE copolymer was 16452, and the polymer dispersity index (PDI) was 1.25.

In order to determine the micelle forming ability of Gln-PEG-b-PAE copolymer, the critical micelle concentration (CMC) of the copolymer was assessed by pyrene fluorescein experiment [24]. The CMC of Gln-PEG- $b$-PAE copolymer was calculated according to the intensity ratio of the first emission peak to the third emission peak I1/I3 (the fluorescence intensity at $373 \mathrm{~nm}$ and at $384 \mathrm{~nm}$ ) plotted against the base 10 logarithm of the tested concentrations $[25,26]$. The result showed that at physiological $\mathrm{pH}$ (7.4), the CMC of Gln-PEG- $b$-PAE copolymer was about $0.016 \mathrm{mg} / \mathrm{mL}$ (see Figure 2(b)), which means that thermodynamically structural stable polymeric micelles had formed under physiological conditions. The low CMC value enables the drug to be stably encapsulated in the micellar systems, so as to improve the bioavailability of the drug [27]. In this study, under physiological environment ( $\mathrm{pH} 7.4)$, with the CMC value being of ca. $0.016 \mathrm{mg} / \mathrm{mL}$, Gln-PEG- $b$-PAE copolymer could not only effectively carry DOX but also maintain the stability of the micellar structure. These results indicated that Gln-PEG- $b$-PAE copolymer was successfully prepared and can be used as drug carrier due to the ability of keeping structural stability under physiological conditions.

\subsection{Characterizations of DOX-Loaded Polymeric Micelles.} The morphology of Gln-PEG- $b$-PAE copolymer and DOX-loaded micelles was characterized by dynamic light scattering (DLS) and transmission electron microscopy (TEM). The result of the light scattering experiments showed that the diameter of Gln-PEG- $b$-PAE@DOX micelles was $75.04 \mathrm{~nm}$, which was $10 \mathrm{~nm}$ more than empty Gln-PEG- $b$ PAE copolymer (see Figures 2(d) and 2(e)), indicating that the average size of DOX-loaded polymeric micelles increased due to the entrapped DOX molecules. TEM also showed that the DOX-loaded polymeric micelles were in a spherical morphology (see Figure 2(c)), with an average size of about $45 \mathrm{~nm}$.

These results demonstrated that we have successfully prepared DOX-loaded polymeric micelles and formed a uniformly distributed nanoscaled micelles structure in an aqueous medium. A previous study reported that the size of nanoparticles is one of the keys to determine whether to have an enhanced permeability and retention (EPR) effect, which also could be explained as the ability to accumulate in tumor tissues [28]. In this study, with the average size of about $75.04 \mathrm{~nm}$, Gln-PEG-b-PAE@DOX micelles may show EPR-mediated passive targeting ability to accumulate in tumor tissues.

\subsection{Stability and Drug Release of Gln-PEG-b-PAE@DOX.} The pH-responsive release of DOX from Gln-PEG- $b$-PAE@DOX micelles was detected by the dialysis method in different simulated buffer solutions, including $\mathrm{pH} 7.4$ of the physiological environment and $\mathrm{pH} 6.0$ of tumor tissues. The $\mathrm{pH}$-dependent DOX release profile is shown in Figure 3(a). In the initial 8 hours, DOX was explosively released under $\mathrm{pH}$ of 6.0 , with about $80 \%$ release burst. The fast release rate of DOX indicated that DOX-loaded micelles were rapidly demicellized to release the drug in acidic environments. However, DOX was slowly released from the micelles at $\mathrm{pH}$ 7.4. After the initial burst of about $30 \%$, the cumulative release grew slowly and reached the plateau gradually. Gln-PEG-b-PAE@DOX micelles exhibited significant pHdependent micellization-decellularization behavior, and micelles released DOX rapidly in an acidic environment of pH 6.0 but very slowly under physiological conditions. Moreover, as shown in Figure 3(b), Gln-PEG-b-PAE copolymer and Gln-PEG- $b$-PAE@DOX micelles retained steady sizes in pH7.4 buffer solutions within $24 \mathrm{~h}$. It is reasonable to believe that the Gln-PEG-b-PAE@DOX micelles can maintain structural integrity for a sufficient time before being distributed to the tumor tissues.

The key to the successful design of drug-loaded polymer micelles is to be able to control the location of drug release. Previous studies have revealed that $\mathrm{pH}$-responsive polymer micelles allow the drug to be efficiently released in the acidic environment of tumor tissues $[29,30]$. Consistently, in this study, Gln-PEG-b-PAE@DOX micelles effectively released DOX under acidic conditions, showing significant $\mathrm{pH}$-dependent micellization-decellularization behavior. Importantly, Gln-PEG- $b$-PAE@DOX micelles could reduce the release of DOX at physiological $\mathrm{pH}$ conditions, so as to reduce the side effects. Moreover, in this study, Gln-PEG- $b$ PAE@DOX micelles retained steady sizes in pH 7.4 buffer solutions within $24 \mathrm{~h}$. It is reasonable to believe that Gln-PEG-b-PAE@DOX micelles could maintain structural stability in physiological environment during drug release, ensuring the feasibility of Gln-PEG- $b$-PAE copolymer as drug carrier.

3.5. In Vitro Cellular Affections. MTT assay and flow cytometry were carried out, in order to verify the antiproliferative and apoptosis effects of Gln-PEG- $b$-PAE@DOX micelles on MDA-MB-231 cells correspondingly. As shown in Figure 4(a), Gln-PEG-b-PAE@DOX micelles showed greater antiproliferative activity compared to free DOX. It could be explained that compared to cells treated with free DOX, cells treated with Gln-PEG- $b$-PAE@DOX micelles were effectively targeted, which led to stronger antiproliferative activity. Additionally, Gln-PEG- $b$-PAE copolymer 


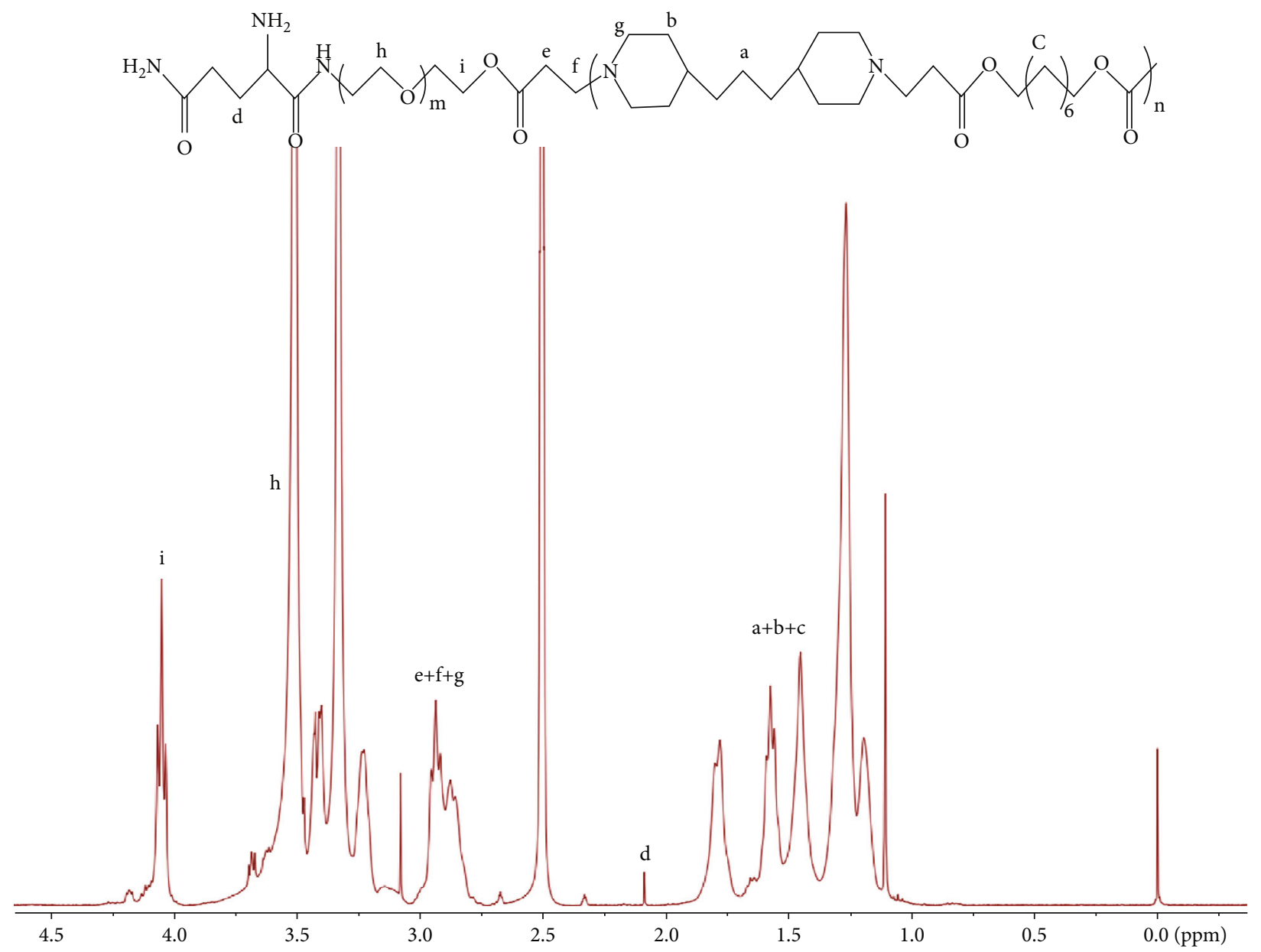

(a)

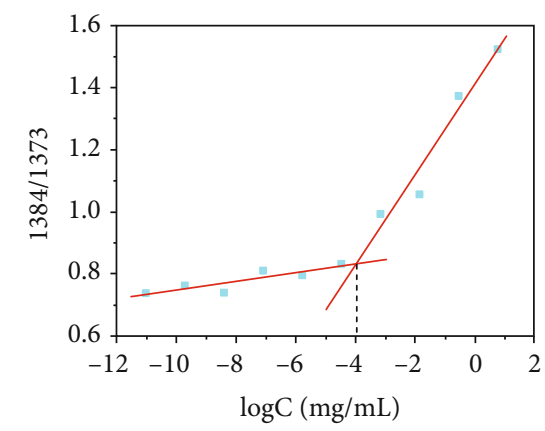

(b)

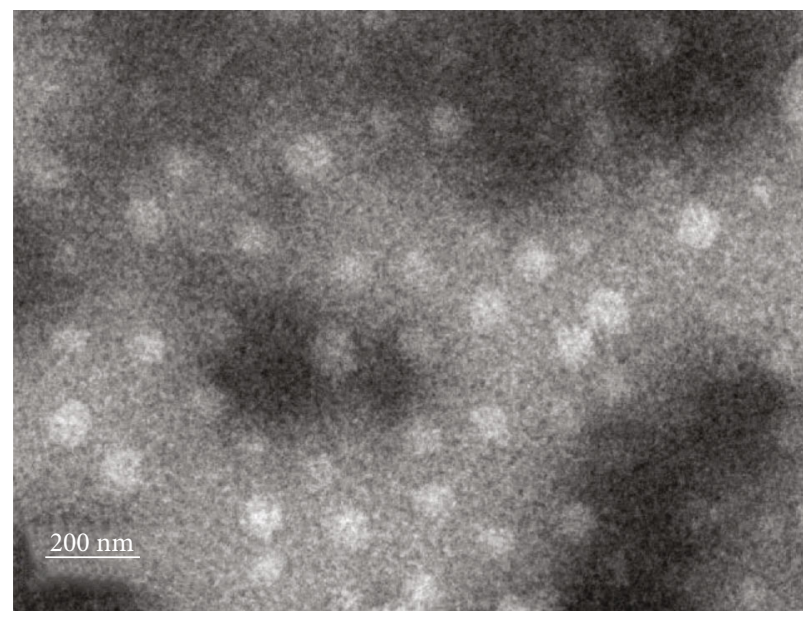

(c)

Figure 2: Continued. 


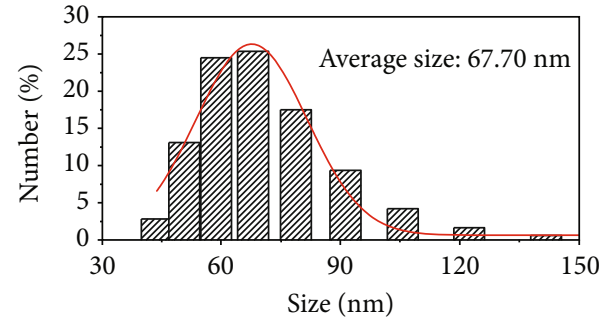

(d)

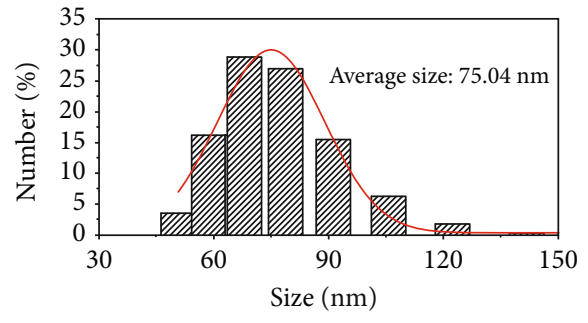

(e)

Figure 2: Characterization of the obtained copolymers. (a) ${ }^{1} \mathrm{H}$ NMR spectrum of Gln-PEG- $b$-PAE copolymer. (b) The critical micelle concentration (CMC) of Gln-PEG- $b$-PAE copolymer is measured by the intensity ratio of the first emission peak to the third emission peak I1/I3 (the fluorescence intensity at $373 \mathrm{~nm}$ and at $384 \mathrm{~nm}$ ). (c) TEM photo of Gln-PEG- $b$-PAE@DOX micelles. Hydrodynamic size distribution profiles of (d) Gln-PEG-b-PAE copolymer and (e) Gln-PEG-b-PAE@DOX micelles determined by dynamic light scattering (DLS).

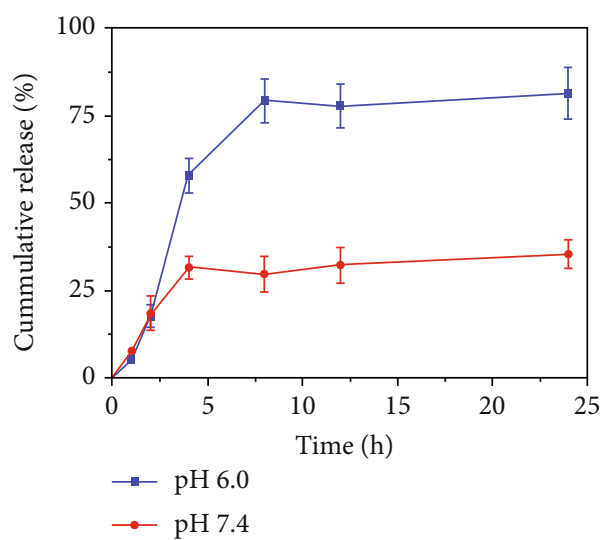

(a)

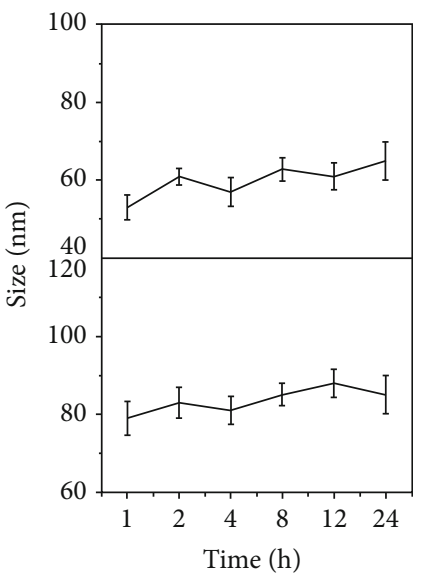

(b)

FIgUre 3: Stability and drug release of the micelles. (a) The cumulative release of DOX from Gln-PEG-b-PAE@DOX micelles at pH 6. 0 (blue line) and 7.4 (red line). (b) The change curve of micelles size vs. time of Gln-PEG- $b$-PAE copolymer (top) and Gln-PEG- $b$ PAE@DOX micelles (bottom).

and mPEG- $b$-PAE copolymer did not significantly inhibit viability of MDA-MB-231 cells, indicating that the GlnPEG- $b$-PAE and mPEG- $b$-PAE copolymers have good biocompatibility to be used as drug carrier. The apoptosis in MDA-MB-231 cells was evaluated after treatment with free DOX and Gln-PEG- $b$-PAE@DOX micelles. As shown in Figure 4(b), the lower- and upper-right quadrants represent the populations of early apoptotic cells and late apoptotic cells, respectively. A $48 \mathrm{~h}$ treatment with Gln-PEG-b-PAE@DOX micelles induced 1.57-fold increased cell death (defined by Annexin $\mathrm{V}$ positivity) compared to the treatment with free DOX. The improved endocytosis resulting from the glutamine ligand of the micelles enhanced the cytotoxicity against MDA-MB-231 cells. It is noteworthy that GlnPEG- $b$-PAE copolymer itself has limited antiproliferative effect on MDA-MB-231 cells. After loading with DOX, Gln-PEG- $b$-PAE micelles showed significantly greater effect on inhibiting cell proliferation and on promoting apoptosis of MDA-MB-231 cells than free DOX. Therefore, Gln-PEG-
$b$-PAE copolymer could be an effective drug carrier with good biocompatibility for TNBC treatment.

Moreover, a glutamine competition experiment was performed to prove whether Gln-PEG-b-PAE@DOX micelles could target MDA-MB-231 cells through SLC1A5 receptormediated transport. As shown in Figures 4(c)-4(e), after coincubation with Gln-PEG- $b$-PAE@DOX micelles for 6 hours, MDA-MB-231 cells absorbed a large amount of DOX released from the micelles; however, the fluorescence intensity of MDA-MB-231 cells treated with Gln-PEG- $b$ PAE@DOX micelles combined with $5 \mathrm{mg} / \mathrm{mL}$ of free glutamine was significantly weaker than that of the group treated only with Gln-PEG- $b$-PAE@DOX micelles. This result showed that free glutamine can compete for the SLC1A5 receptor on MDA-MB-231 cells and reduce the cellular uptake of DOX released from Gln-PEG-b-PAE@DOX micelles at the same time. The glutamine competition experiment has proved that Gln-PEG- $b$-PAE@DOX micelles have the ability to actively target TNBC. With the characteristics 


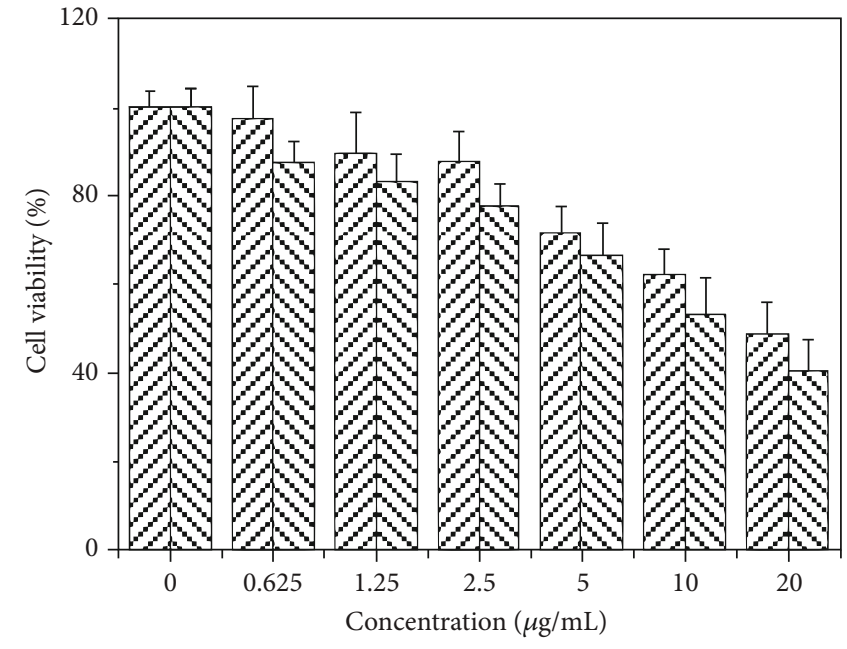

Fas

W Gln-PEG-b-PAE@Dox

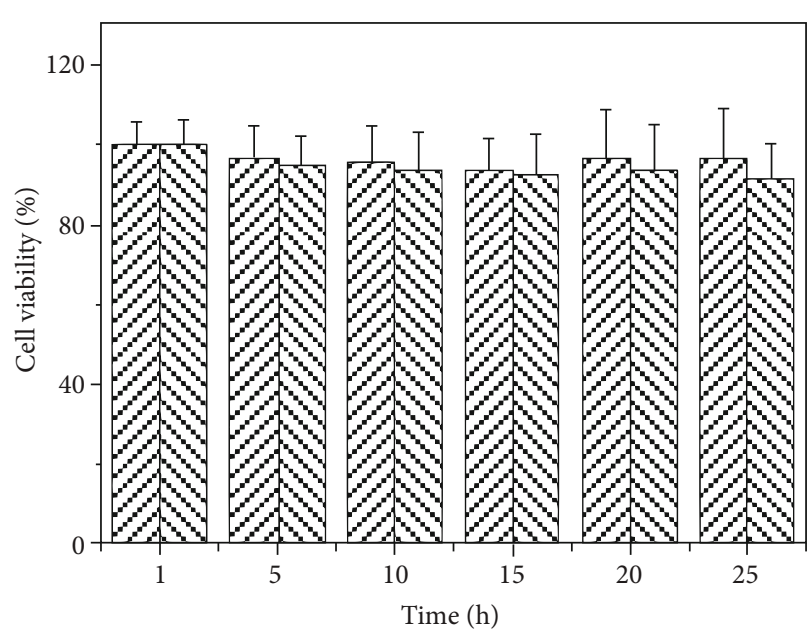

22 PEG-b-PAE

W Gln-PEG- $b$-PAE

(a)

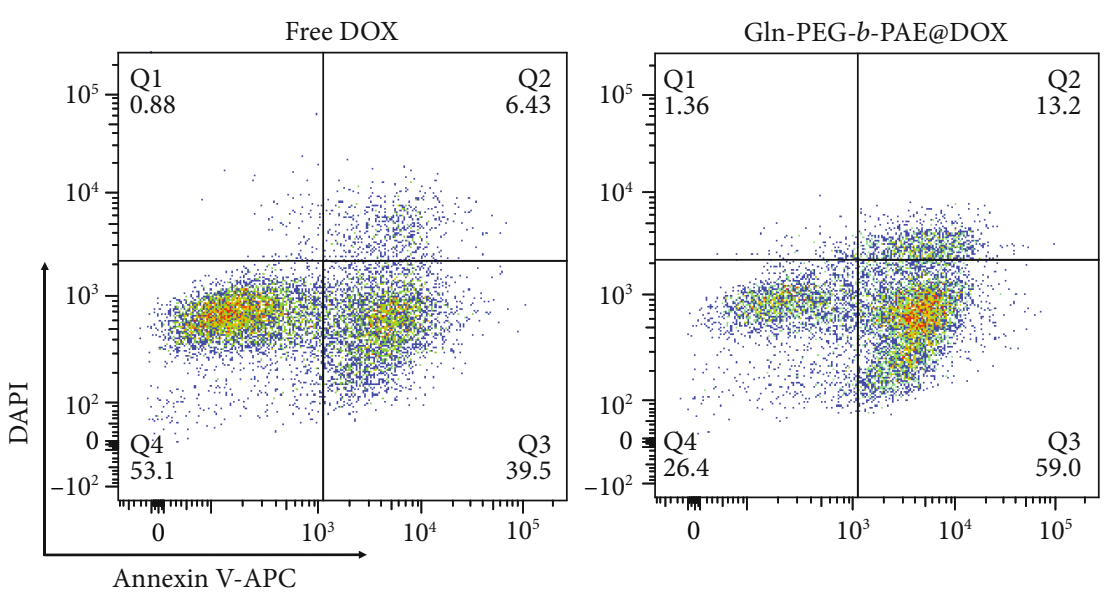

(b)
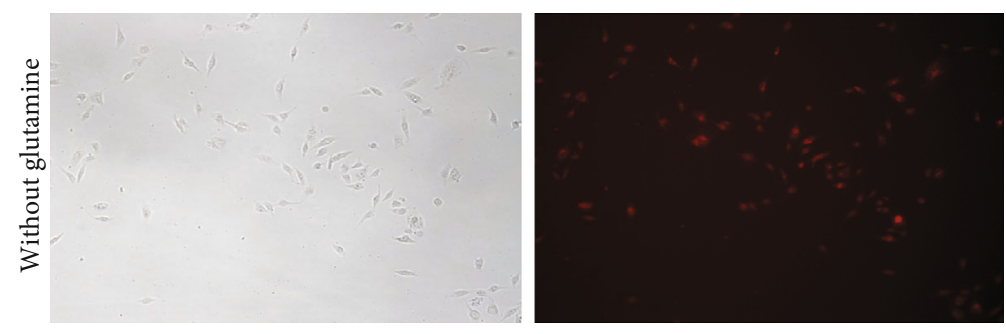

(c)

Figure 4: Continued. 

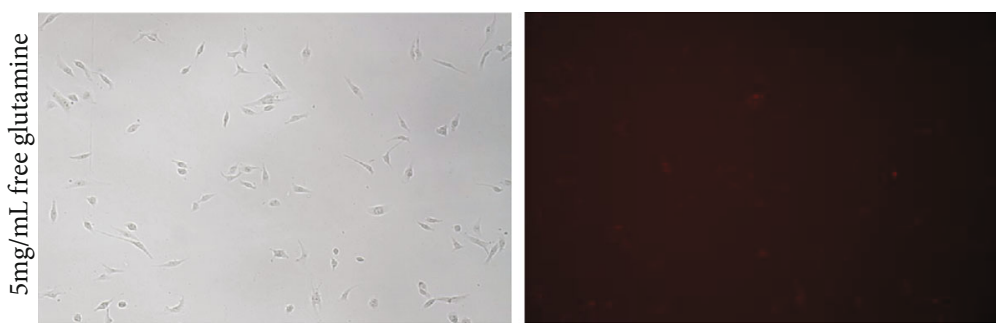

(d)
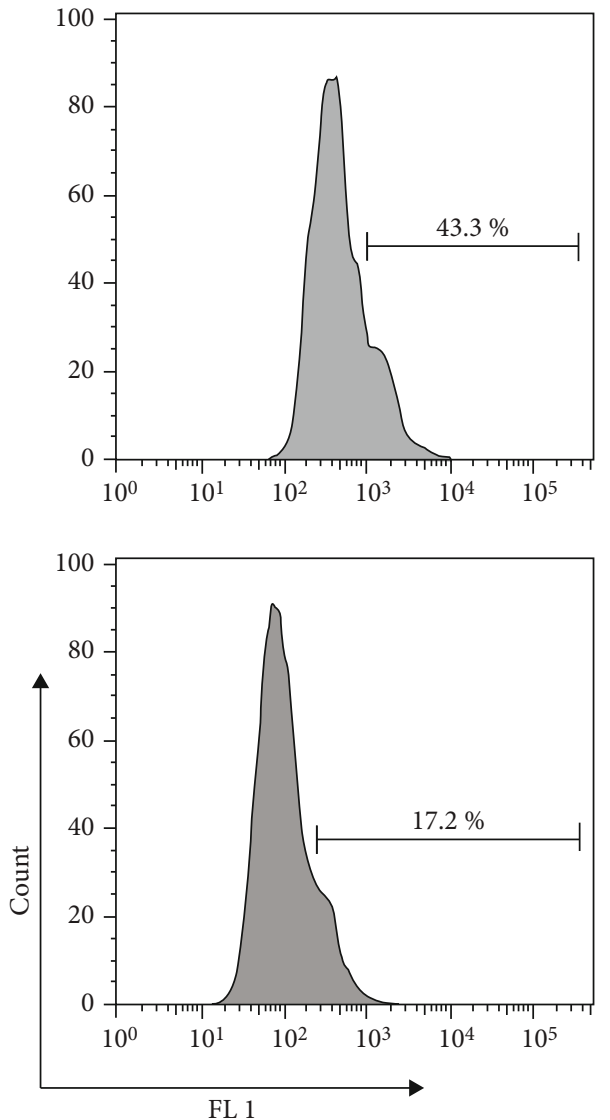

(e)

FIGURE 4: The effect of copolymers and free DOX on MDA-MB-231 cells. (a) Cell viability of MDA-MB-231 cells treated with different concentrations of free DOX and Gln-PEG- $b$-PAE@DOX micelles, respectively (left), and cell viability of MDA-MB-231 cells treated with mPEG- $b$-PAE and Gln-PEG- $b$-PAE copolymers, respectively, at different time checkpoint (right). (b) Cell apoptosis of MDA-MB-231 cells treated with free DOX and Gln-PEG-b-PAE@DOX micelles. In each panel, the lower-left (Annexin $\mathrm{V}^{-}, \mathrm{DAPI}^{-}$), lower-right (Annexin $\mathrm{V}^{+}, \mathrm{DAPI}^{-}$), and upper-right (Annexin $\mathrm{V}^{+}, \mathrm{DAPI}^{+}$) quadrants represent the populations of living cells, apoptotic cells, and necrotic/dead cells, respectively. (c) Intracellular uptake of DOX in MDA-MB-231 incubated with Gln-PEG- $b$-PAE@DOX micelles. (d) Intracellular uptake of DOX in MDA-MB-231 incubated with Gln-PEG-b-PAE@DOX micelles combined with $5 \mathrm{mg} / \mathrm{mL}$ of free glutamine. (e) Intracellular uptake of DOX in MDA-MB-231 incubated with Gln-PEG- $b$-PAE@DOX micelles (up) and Gln-PEG- $b$ PAE@DOX micelles combined with $5 \mathrm{mg} / \mathrm{mL}$ of free glutamine (bottom).

of both pH-responsive release of DOX and glutaminemediated active targeting ability, Gln-PEG- $b$-PAE@DOX micelles could be a potential TNBC-targeted drug delivery system.

3.6. In Vivo Antitumor Efficacy of Gln-PEG-b-PAE@DOX Micelles. The antitumor efficacy of free DOX, mPEG- $b$ PAE@DOX, and Gln-PEG- $b$-PAE@DOX micelles in vivo was evaluated in tumor-bearing $\mathrm{BLAB} / \mathrm{c} \mathrm{nu} / \mathrm{nu}$ mice (see Figure 5(a)). When the mice were treated with Gln-PEG- $b$-PAE@DOX micelles, the tumor growth rate was significantly slower compared to those treated with free DOX and saline (see Figure 5(b)). After 17 days, free DOX at $2 \mathrm{mg} / \mathrm{kg}$ suppressed tumor volumes by $45.6 \%$, compared to the control group, while mPEG- $b$-PAE@DOX micelles at $2 \mathrm{mg} \mathrm{DOX} / \mathrm{kg}$ inhibited tumor sizes by $63.7 \%$. Importantly, Gln-PEG- $b$-PAE@DOX micelles at $2 \mathrm{mg}$ DOX $/ \mathrm{kg}$ significantly inhibited tumor sizes by 78.3\% (see Figures 5(d)-5(f)). Additionally, compared with mice treated with saline and free DOX, mice treated with Gln-PEG- $b$-PAE@DOX micelles had significantly lower fluorescence intensity (see Figures 5(c) and 5(g)). Tumor volumes in mice treated with mPEG-b-PAE@DOX micelles were significantly smaller than those in mice treated with free DOX. This result could be explained by the EPR effect which makes mPEG- $b$-PAE@DOX micelles more likely to be accumulated in tumors. Previous studies have also reported this passive accumulation of polymer conjugate in various solid tumor models due to the EPR effect $[28,31,32]$. Generally, in this study, Gln-PEG-b-PAE@DOX micelles showed the highest antitumor effect on transplanted tumors compared with mPEG- $b$-PAE@DOX micelles and free DOX. The pHdependent release of DOX and glutamine-mediated targeting ability make Gln-PEG- $b$-PAE@DOX micelles show excellent antitumor effect not only in vitro but also in vivo. Therefore, it is reasonable to believe Gln-PEG- $b$ PAE@DOX micelles are an ideal tumor-targeting drug delivery system for TNBC. 


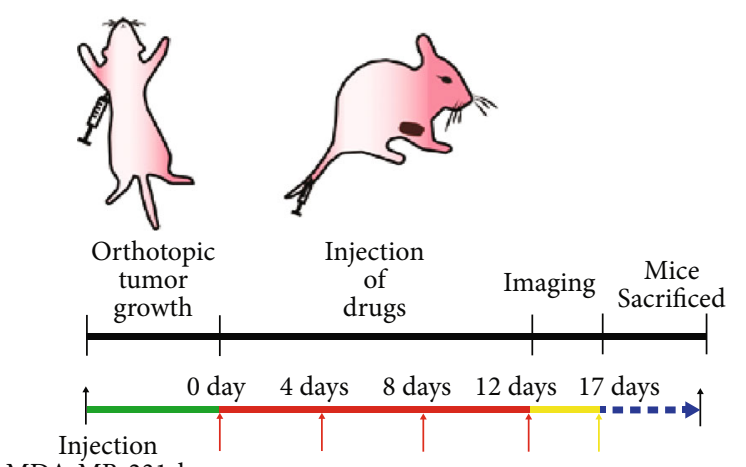

MDA-MB-231-luc

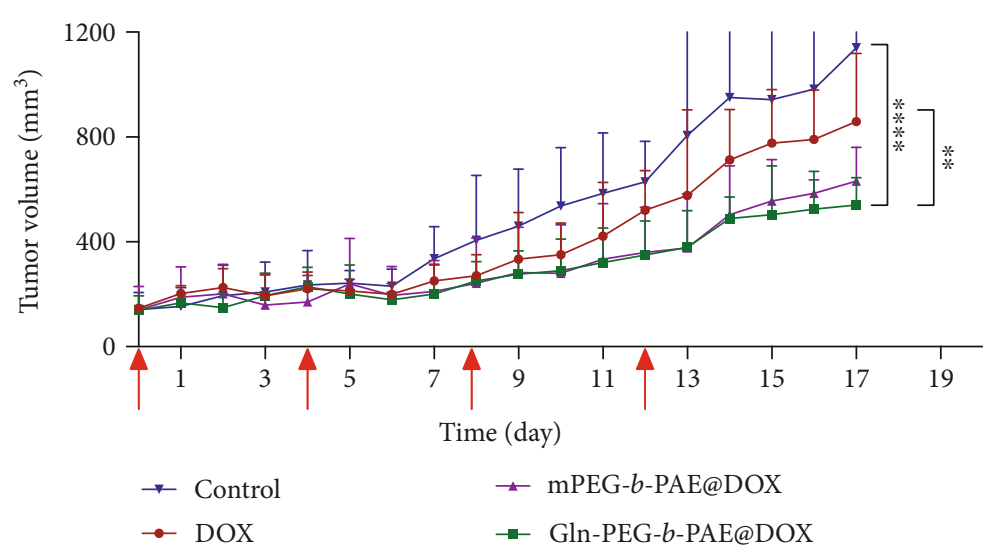

(b)
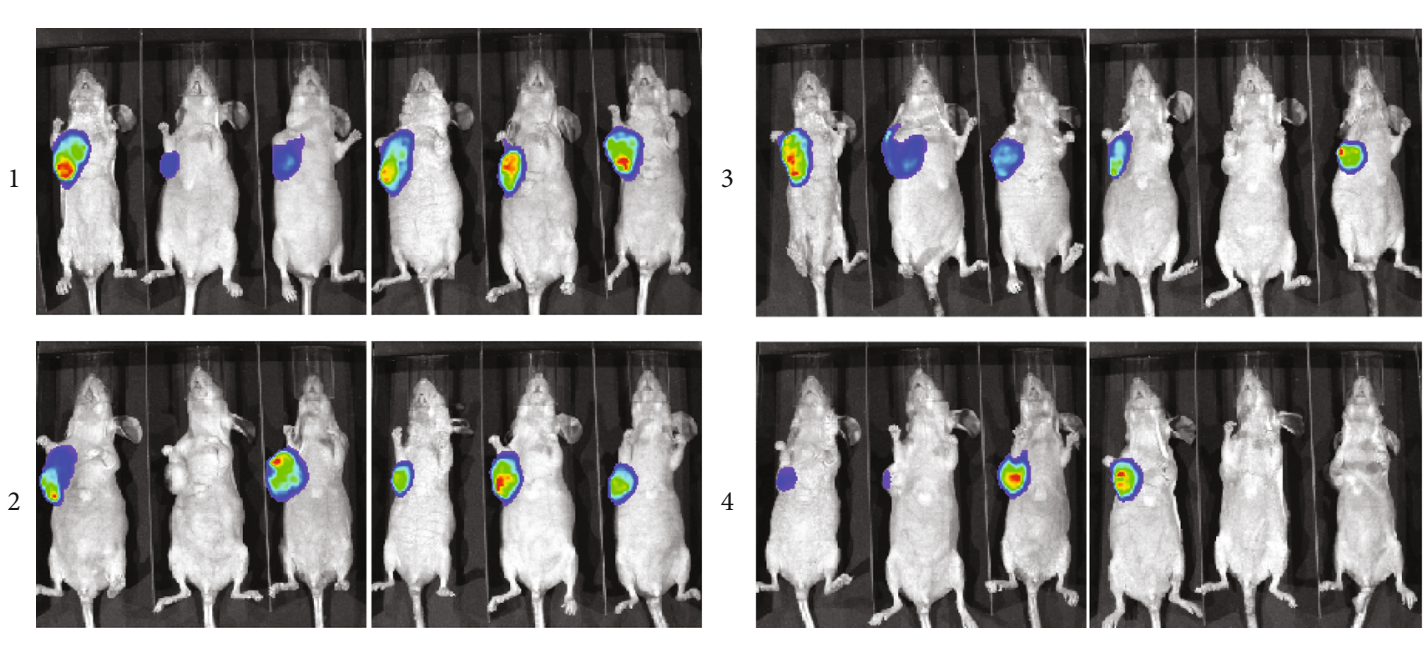

(c)

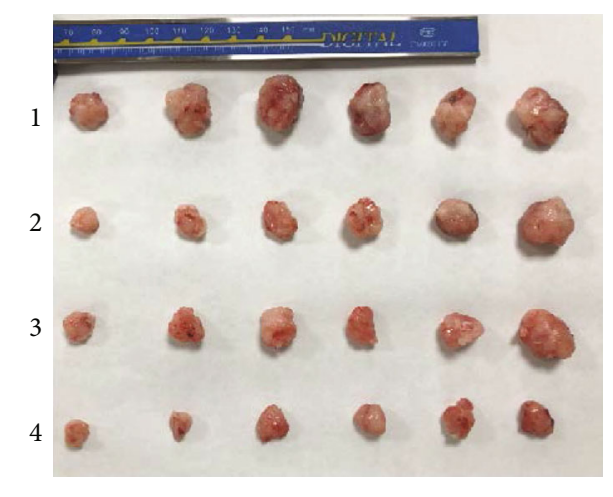

(d)

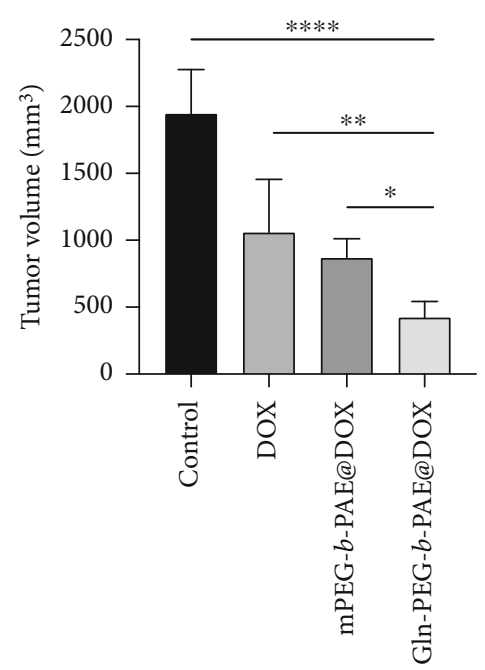

(e)

Figure 5: Continued. 


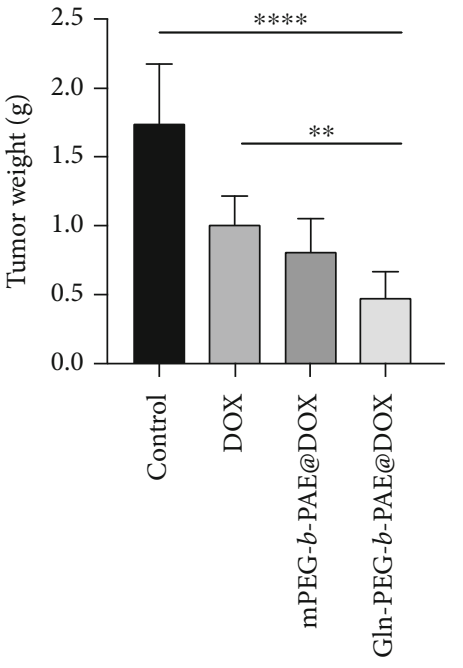

(f)

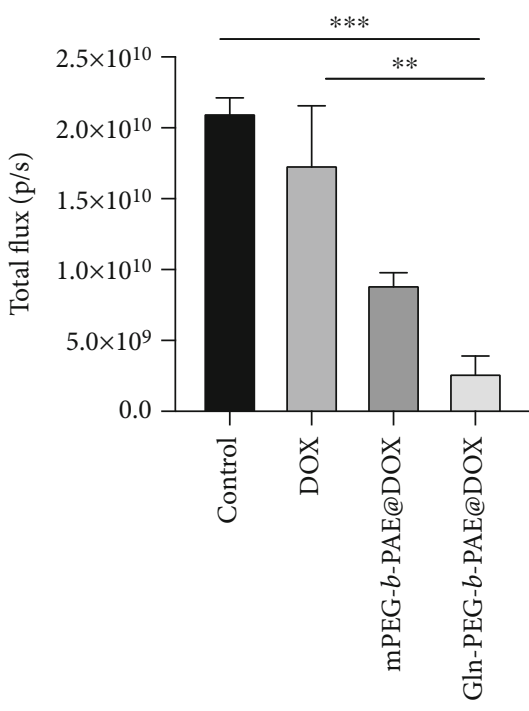

(g)

FIgURE 5: Antitumor efficacy of Gln-PEG-b-PAE@DOX in vivo. (a) Schematic illustration of the in vivo experiment. (b) Tumor growth curves of tumor-bearing mice treated with saline, DOX, mPEG- $b$-PAE@DOX, and Gln-PEG- $b$-PAE@DOX. (c, g) Fluorescence photo and total flux of mice treated with saline (1), DOX (2), mPEG- $b$-PAE@DOX (3), and Gln-PEG-b-PAE@DOX (4). (d) Photographs of dissected tumors of the control group (1), free DOX group (2), mPEG- $b$-PAE@DOX group (3), and Gln-PEG- $b$-PAE@DOX group (4). (e, f) Volume and weight of dissected tumors. Comparisons between the groups were performed using one-way analysis of variance (ANOVA); ${ }^{*} p<0.05,{ }^{* *} p<0.01$, ${ }^{* * *} p<0.001$, and ${ }^{* * * *} p<0.0001$.

\section{Conclusions}

In this study, glutamine was conjugated to the amphiphilic PEG- $b$-PAE copolymer, in which glutamine was used for active targeting of TNBC cells. The pH-triggered Gln-PEG$b$-PAE@DOX copolymeric micelles showed a spherical morphology with an average size about $75.04 \mathrm{~nm}$, being able to maintain structural stability in physiological environment and release DOX in tumoral acidic environment effectively. In vitro, Gln-PEG- $b$-PAE copolymer showed low cytotoxicity, while Gln-PEG- $b$-PAE@DOX micelles showed significantly stronger cytotoxicity. Compared to free DOX and mPEG- $b$-PAE@DOX, Gln-PEG- $b$-PAE@DOX micelles exhibited significantly higher ability to inhibit tumor growth in vivo due to $\mathrm{pH}$-responsive release of DOX and glutaminemediated active tumor-targeting ability. Therefore, the amphiphilic Gln-PEG- $b$-PAE@DOX micelles may be a new target therapy strategy for TNBC, and also, Gln-PEG- $b$ PAE copolymer could be a promising carrier of antitumor drugs for TNBC.

\section{Data Availability}

The datasets used and/or analyzed during the current study are available from the corresponding authors on reasonable request.

\section{Conflicts of Interest}

The authors declare that there is no conflict of interest regarding the publication of this paper.

\section{Authors' Contributions}

Yi-Zhi Zhu and Di Xu contributed equally to this work.

\section{Acknowledgments}

We thank Chen-Xi Chen from the Analysis Center of Nanjing Medical University for his technical support with flow cytometry in apoptosis assay. This work was supported by the National Natural Science Foundation of China (grant numbers 81872365, 81801827, and 81901883) and the Basic Research Program of Jiangsu Province (grant numbers BK20181086 and BK20191080).

\section{References}

[1] C. D. Aluise, R. Sultana, J. Tangpong et al., "Chemo brain (chemo fog) as a potential side effect of doxorubicin administration: role of cytokine-induced, oxidative/nitrosative stress in cognitive dysfunction," Advances in Experimental Medicine and Biology, vol. 678, pp. 147-156, 2010.

[2] C. Carvalho, R. Santos, S. Cardoso et al., "Doxorubicin: the good, the bad and the ugly effect," Current Medicinal Chemistry, vol. 16, no. 25, pp. 3267-3285, 2009.

[3] F. Fan, L. Wang, Z. Ouyang, Y. Wen, and X. Lu, "Development and optimization of a tumor targeting system based on microbial synthesized PHA biopolymers and PhaP mediated functional modification," Applied Microbiology and Biotechnology, vol. 102, no. 7, pp. 3229-3241, 2018.

[4] E. Perez-Herrero and A. Fernandez-Medarde, "Advanced targeted therapies in cancer: drug nanocarriers, the future of chemotherapy," European Journal of Pharmaceutics and Biopharmaceutics, vol. 93, pp. 52-79, 2015. 
[5] Y. F. Ding, J. Wei, S. Li, Y. T. Pan, L. H. Wang, and R. Wang, "Host-guest interactions initiated supramolecular chitosan nanogels for selective intracellular drug delivery," ACS Applied Materials \& Interfaces, vol. 11, no. 32, pp. 28665-28670, 2019.

[6] Y. F. Ding, S. Li, L. Liang et al., "Highly biocompatible chlorin e6-loaded chitosan nanoparticles for improved photodynamic cancer therapy," ACS Applied Materials \& Interfaces, vol. 10, no. 12, pp. 9980-9987, 2018.

[7] C. Gao, F. Tang, G. Gong et al., "pH-responsive prodrug nanoparticles based on a sodium alginate derivative for selective co-release of doxorubicin and curcumin into tumor cells," Nanoscale, vol. 9, no. 34, pp. 12533-12542, 2017.

[8] Y. Huang, C. Song, H. Li et al., "Cationic conjugated polymer/hyaluronan-doxorubicin complex for sensitive fluorescence detection of hyaluronidase and tumor-targeting drug delivery and imaging," ACS Applied Materials \& Interfaces, vol. 7, no. 38, pp. 21529-21537, 2015.

[9] Y. Bae, N. Nishiyama, S. Fukushima, H. Koyama, M. Yasuhiro, and K. Kataoka, "Preparation and biological characterization of polymeric micelle drug carriers with intracellular $\mathrm{pH}$-triggered drug release property: tumor permeability, controlled subcellular drug distribution, and enhanced in vivo antitumor efficacy," Bioconjugate Chemistry, vol. 16, no. 1, pp. 122-130, 2005.

[10] Y. Bae and K. Kataoka, "Significant enhancement of antitumor activity and bioavailability of intracellular $\mathrm{pH}$-sensitive polymeric micelles by folate conjugation," Journal of Controlled Release, vol. 116, no. 2, pp. e49-e50, 2006.

[11] Y. Miura, T. Takenaka, K. Toh et al., "Cyclic RGD-linked polymeric micelles for targeted delivery of platinum anticancer drugs to glioblastoma through the blood-brain tumor barrier," ACS Nano, vol. 7, no. 10, pp. 8583-8592, 2013.

[12] Y. Zhao, Y. Zhou, D. Wang et al., "pH-responsive polymeric micelles based on poly(2-ethyl-2-oxazoline)-poly(D,L-lactide) for tumor-targeting and controlled delivery of doxorubicin and P-glycoprotein inhibitor," Acta Biomaterialia, vol. 17, pp. 182-192, 2015.

[13] M. Huo, A. Zou, C. Yao et al., "Somatostatin receptormediated tumor-targeting drug delivery using octreotidePEG-deoxycholic acid conjugate-modified N-deoxycholic acid-O, N-hydroxyethylation chitosan micelles," Biomaterials, vol. 33, no. 27, pp. 6393-6407, 2012.

[14] B. Godin, E. Tasciotti, X. Liu, R. E. Serda, and M. Ferrari, "Multistage nanovectors: from concept to novel imaging contrast agents and therapeutics," Accounts of Chemical Research, vol. 44, no. 10, pp. 979-989, 2011.

[15] M. I. Gross, S. D. Demo, J. B. Dennison et al., "Antitumor activity of the glutaminase inhibitor CB-839 in triplenegative breast cancer," Molecular Cancer Therapeutics, vol. 13, no. 4, pp. 890-901, 2014.

[16] S. Kim, D. H. Kim, W. H. Jung, and J. S. Koo, "Expression of glutamine metabolism-related proteins according to molecular subtype of breast cancer," Endocrine-Related Cancer, vol. 20, no. 3, pp. 339-348, 2013.

[17] M. van Geldermalsen, Q. Wang, R. Nagarajah et al., "ASCT2/SLC1A5 controls glutamine uptake and tumour growth in triple-negative basal-like breast cancer," Oncogene, vol. 35, no. 24, pp. 3201-3208, 2016.

[18] F. Oppedisano, L. Pochini, M. Galluccio, and C. Indiveri, "The glutamine/amino acid transporter (ASCT2) reconstituted in liposomes: transport mechanism, regulation by ATP and characterization of the glutamine/glutamate antiport," Biochimica et Biophysica Acta, vol. 1768, no. 2, pp. 291-298, 2007.
[19] H. Chen, Y. Chen, H. Yang et al., “A dual-targeting nanocarrier based on modified chitosan micelles for tumor imaging and therapy," Polymer Chemistry, vol. 5, no. 16, p. 4734, 2014.

[20] M. L. Schulte, A. Fu, P. Zhao et al., "Pharmacological blockade of ASCT2-dependent glutamine transport leads to antitumor efficacy in preclinical models," Nature Medicine, vol. 24, no. 2, pp. 194-202, 2018.

[21] C. Wang, J. Wu, Z. Wang et al., "Glutamine addiction activates polyglutamine-based nanocarriers delivering therapeutic siRNAs to orthotopic lung tumor mediated by glutamine transporter SLC1A5," Biomaterials, vol. 183, pp. 77-92, 2018.

[22] M. S. Kim, S. J. Hwang, J. K. Han et al., "pH-responsive PEGpoly $(\beta$-amino ester) block copolymer micelles with a sharp transition," Macromolecular Rapid Communications, vol. 27, no. 6, pp. 447-451, 2006.

[23] S. M. Lee, R. W. Ahn, F. Chen et al., "Biological evaluation of $\mathrm{pH}$-responsive polymer-caged nanobins for breast cancer therapy," ACS Nano, vol. 4, no. 9, pp. 4971-4978, 2010.

[24] Z. Duan, Y. J. Gao, Z. Y. Qiao et al., "pH-sensitive polymer assisted self-aggregation of bis(pyrene) in living cells in situ with turn-on fluorescence," Nanotechnology, vol. 26, no. 35, p. 355703, 2015.

[25] J. Z. Du, D. P. Chen, Y. C. Wang et al., "Synthesis and micellization of amphiphilic brush-coil block copolymer based on poly(epsilon-caprolactone) and PEGylated polyphosphoester," Biomacromolecules, vol. 7, no. 6, pp. 1898-1903, 2006.

[26] X. Gao, B. Wang, X. Wei et al., "Preparation, characterization and application of star-shaped PCL/PEG micelles for the delivery of doxorubicin in the treatment of colon cancer," International Journal of Nanomedicine, vol. 8, pp. 971-982, 2013.

[27] Y. Lu, Z. Yue, J. Xie et al., "Micelles with ultralow critical micelle concentration as carriers for drug delivery," Nature Biomedical Engineering, vol. 2, no. 5, pp. 318-325, 2018.

[28] H. Maeda, "Polymer therapeutics and the EPR effect," Journal of Drug Targeting, vol. 25, no. 9-10, pp. 781-785, 2017.

[29] E. R. Gillies and J. M. J. Frechet, "pH-responsive copolymer assemblies for controlled release of doxorubicin," Bioconjugate Chemistry, vol. 16, no. 2, pp. 361-368, 2005.

[30] X. Huang, W. Liao, G. Zhang, S. Kang, and C. Y. Zhang, "pHsensitive micelles self-assembled from polymer brush (PAE- $g$ cholesterol)- $b$-PEG- $b$-(PAE- $g$-cholesterol) for anticancer drug delivery and controlled release," International Journal of Nanomedicine, vol. 12, pp. 2215-2226, 2017.

[31] H. Maeda, "SMANCS and polymer-conjugated macromolecular drugs: advantages in cancer chemotherapy," Advanced Drug Delivery Reviews, vol. 46, no. 1-3, pp. 169-185, 2001.

[32] L. W. Seymour, Y. Miyamoto, H. Maeda et al., "Influence of molecular weight on passive tumour accumulation of a soluble macromolecular drug carrier," European Journal of Cancer, vol. 31A, no. 5, pp. 766-770, 1995. 